\title{
Chemotherapy induces ovarian cancer cell repopulation through the caspase 3-mediated arachidonic acid metabolic pathway
}

This article was published in the following Dove Press journal:

OncoTargets and Therapy

\author{
Lianzhi Cui ${ }^{1,2}$ \\ Yawei Zhao' \\ Yue Pan' \\ Xiao Zheng' \\ Dan Shao' \\ Yong Jia ${ }^{3}$ \\ Kan $\mathrm{He}^{1}$ \\ Kun $\mathrm{Li}^{3}$ \\ Li Chen ${ }^{1,3}$ \\ 'Department of Pharmacology, \\ College of Basic Medical Sciences, \\ Jilin University, Changchun, ${ }^{2}$ Clinical \\ Laboratory, Jilin Cancer Hospital, \\ Changchun, ${ }^{3}$ School of Nursing, Jilin \\ University, Changchun, China
}

Correspondence: Li Chen Department of Pharmacology, College of Basic Medical Sciences, Jilin University, I26 Xinmin Street, Changchun I3002I, Jilin, China

Email chenl@jlu.edu.cn

Kun Li

School of Nursing, Jilin University, 965 Xinjiang Street, Changchun I3002I, Jilin, China

Email lik@jlu.edu.cn

\begin{abstract}
Recurrence is one of the major causes of high mortality in ovarian cancer. However, the mechanism of ovarian cancer recurrence after chemotherapy has not been fully understood. In the present study, we investigated the effect of chemotherapy-induced tumor microenvironment on the proliferation of SKOV3 cells. We have shown that SKOV3 cells repopulated faster in the culture medium from apoptotic SKOV3 ovarian cancer cells after $24 \mathrm{~h}$ of etoposide phosphate (VP-16) treatment. We found that during apoptosis, cleaved caspase 3 could activate cytosolic calcium-independent phospholipase $\mathrm{A}_{2}$, which stimulated the release of arachidonic acid (AA) and triggered the production of prostaglandin $\mathrm{E}_{2}\left(\mathrm{PGE}_{2}\right)$. An increased level of phosphorylated focal adhesion kinase (FAK) subsequently facilitated the reproliferation of SKOV3 cells, and VP-16-induced repopulation effects were partially reversed by the FAK inhibitor PF562271. Furthermore, the plasma AA-to- $\mathrm{PGE}_{2}$ ratio and tumoral FAK expression of ovarian cancer patients after chemotherapy were significantly lower than those before chemotherapy. Taken together, our results indicate that chemotherapy-induced apoptotic cancer cells can produce $\mathrm{PGE}_{2}$-enriched microenvironment through caspase 3-mediated AA metabolic pathway, which could lead to the abnormal activation of FAK and eventually accelerate the repopulation of SKOV3 cells. Our study provides novel insight into a mechanism that may be utilized to prevent ovarian cancer recurrence in response to chemotherapy.
\end{abstract}

Keywords: ovarian cancer, repopulation, chemotherapy, apoptosis, FAK

\section{Introduction}

Ovarian cancer is the fourth most common gynecological malignancy, the sixth leading cause of cancer deaths among women, and the second leading cause of gynecological malignancy-related deaths worldwide. ${ }^{1}$ According to the National Comprehensive Cancer Network (NCCN) guidelines of 2016, the treatment of women who have advanced or poor-prognosis early-stage ovarian cancer mainly consists of surgery followed by chemotherapy. ${ }^{2}$ Although overall tumor response rates associated with paclitaxel and platinum range from $70 \%$ to $80 \%,{ }^{3} 50 \%-75 \%$ of individuals relapse within 18 months after completing first-line therapy and require further systemic therapy. ${ }^{4}$ Patients who relapse are rarely curable and often have only a short-term progression-free survival. Since apoptosis is a major mechanism of cytotoxin-based chemotherapy, there may be a link between the apoptosis-induced death of tumor cells and the repopulation of surviving cells in ovarian cancer; however, investigations of a specific mechanism for this link are scarce. Thus, we focused on the mechanism of the repopulation of ovarian cancer cells after chemotherapy aiming to provide a novel 
strategy for solving the problem of the high rate of ovarian cancer recrudescence and to improve the treatment and prevention of ovarian cancer.

Several studies have recently focused on the adverse effects of chemotherapy. ${ }^{5-7}$ These findings have demonstrated that induction chemotherapy results in an accelerated repopulation and measurable regrowth of non-small-cell lung cancer tumors within weeks, which is much faster than the reported rates in preclinical models or untreated tumors. The cellular response to chemotherapy is presumed to induce the phenomenon of accelerated repopulation, which is one of the major causes of cancer recurrence. ${ }^{7}$ Therefore, chemotherapy itself may be one of the reasons for cancer relapse, and the source of the repopulating cells constitutes those that survived after the first period of chemotherapy administration. In cancer treatment, apoptosis is a well-recognized cell death mechanism through which cytotoxic agents kill tumor cells. Caspase 3 is a protease that is activated during the execution phase of apoptosis, which contributes to cell death. Cleaved caspase 3 can activate cytosolic calcium-independent phospholipase $\mathrm{A}_{2}\left(\mathrm{iPLA}_{2}\right),{ }^{8}$ and a consequence of membrane phospholipase $\mathrm{A}_{2}\left(\mathrm{PLA}_{2}\right)$ activation is the release of arachidonic acid (AA) from membrane phospholipids, which ultimately triggers production of the growth signal prostaglandin $\mathrm{E}_{2}\left(\mathrm{PGE}_{2}\right){ }^{7}$ Both $\mathrm{AA}$ and $\mathrm{PGE}_{2}$ stimulate cell proliferation and tumor growth. ${ }^{9}$ Thus, caspase 3 activation may also ultimately lead to cancer recrudescence through the AA metabolic pathway. This mechanism has been observed in several different human cancers, but whether and why the activation of the AA metabolic pathway is linked with tumor recrudescence in ovarian cancer is unknown.

Focal adhesion kinase (FAK), a $125-\mathrm{kDa}$ non-receptor cytoplasmic protein tyrosine kinase, is a key regulator of signals from the extracellular matrix mediated by integrins and growth factor receptors. ${ }^{10,11}$ In addition, elevated expression of FAK has been correlated with poor survival rates. FAK has been implicated in the regulation of a variety of cellular signaling pathways that control cell proliferation, cell-cycle progression, migration, apoptosis, and cell survival. ${ }^{11,12}$ In addition, the effects of $\mathrm{PGE}_{2}$ are associated with FAK. $\mathrm{PGE}_{2}$ treatment increases the phosphorylation of FAK in hepatocellular carcinoma (HCC) cells. ${ }^{13}$ However, whether the chemotherapy-induced repopulation of ovarian cancer cells is through the activation of FAK remains unclear. Thus, we hypothesize that FAK may be downstream of the caspaseactivated $\mathrm{iPLA}_{2}-\mathrm{AA}_{-} \mathrm{PGE}_{2}$ axis in the chemotherapy-induced repopulation of ovarian cancer cells.
Herein, we first studied the levels of $\mathrm{PGE}_{2}$ and $\mathrm{AA}$ and the expression of FAK and phosphorylated FAK (p-FAK) in clinical samples. Next, we established a Transwell system for the co-culture of SKOV3 cells with the chemotherapy drug etoposide phosphate (VP-16) to mimic the microenvironmental changes in the dying cells and to stimulate the growth of a small number of surviving ovarian cancer cells. Our results test the hypotheses that the VP-16 treatment triggers apoptosis, caspase 3 cleavage, and activation of $\mathrm{iPLA}_{2}$, stimulating the release of AA and the production of $\mathrm{PGE}_{2}$, resulting in the activation of FAK. The present study indicates a novel mechanism by which chemotherapy promotes ovarian cancer cells repopulation and provides an attractive target for prevention of recurrence.

\section{Materials and methods Chemicals and reagents}

VP-16 was purchased from Qilu Pharmaceutical Co., LTD (Jinan, China). A human $\mathrm{PGE}_{2}$ enzyme-linked immunosorbent assay (ELISA) kit and human AA ELISA kit were purchased from R\&D Systems. PF562271 was purchased from Selleckchem. RPMI 1640 medium, fetal bovine serum (FBS), penicillin, streptomycin, and bicinchoninic acid (BCA) protein assay kits were purchased from Beyotime Institute of Biotechnology (Haimen, China). The primary antibodies, including anti-FAK (Cat. \# ab40794; Abcam), anti-p-FAK (phosphor Tyr397, Cat. \# ab39967; Abcam), anti-iPLA (Cat. \# sc-25504; Santa Cruz Biotechnology), anti-caspase 3 (Cat. \# 836; Cell Signaling), and anti- $\beta$-actin (Cat. \# 1854; Epitomics), were diluted at 1:800 or $1: 1,000$ before use. All chemical compounds were analytically pure reagents.

\section{Clinical samples}

The tissue samples and plasma samples from ovarian cancer patients were collected during routine therapeutic surgery before and after chemotherapy in our hospital. Tissue and plasma samples of patients (stages I and II, n=14) who underwent surgery without chemotherapy were presented as the control group, whereas tissue and plasma samples collected from patients (stages III and IV, $n=12$ ) who received two to four short-course regimens of chemotherapy (2-4 months) were presented as the chemotherapy group. All tissues and plasma samples were immediately frozen in liquid nitrogen after explantation. This work was approved by the Ethics Committee of Jilin Cancer Hospital. All patients gave written informed consent before participating in this study. 


\section{Immunohistochemistry}

Immunohistochemical staining for FAK and p-FAK in the aforementioned patient tissue samples was performed. Briefly, after deparaffinization and blocking, the sections were incubated overnight at $4{ }^{\circ} \mathrm{C}$ with the anti-FAK or antip-FAK antibody. The sections were counterstained with Meyer's hematoxylin. FAK- and p-FAK-positive cells were defined as those with brown staining in the nucleus and cytoplasm, respectively. Signals in tumor tissue of each group were visually quantified using the same scoring system (0-9), multiplied intensity of signal, and percentage of positive cells ( signal: $0=$ no signal, $1=$ weak signal, $2=$ intermediate signal, and $3=$ strong signal; percentage: $0=0 \%, 1=25 \%$, $2=25 \%-50 \%$, and $3=50 \%)^{14}$

\section{Co-culture system and cell proliferation assays}

The human epithelial ovarian cancer cell line SKOV3 was purchased from ATCC and cultured in RPMI 1640 supplemented with $10 \% \mathrm{FBS}$, penicillin (100 units $/ \mathrm{mL}$ ), and streptomycin $(100 \mathrm{mg} / \mathrm{mL})$. Cells were incubated at $37^{\circ} \mathrm{C}$ under $5 \%$ $\mathrm{CO}_{2}$. Six-well Transwell chambers (Corning, Corning, NY, USA) with $4.0 \mu \mathrm{m}$ pore size semipermeable membranes were used to prepare the Transwell-based co-culture system..$^{14,15}$ In the bottom chamber, SKOV3 cells $\left(10^{5}\right.$ cells per well) as the feeder cells were treated with VP-16, and in the upper chamber, fewer numbers of SKOV 3 cells ( 1,000 cells per well) were designed as the receptor cells exposed to culture medium from the feeder cells. In the model group, the feeder cells were rinsed with PBS twice after a $24 \mathrm{~h}$ treatment with $5 \mu \mathrm{M}$ VP-16, fresh culture medium was added, and the SKOV3 receptor cells were inserted into the upper chamber. Meanwhile, a group of feeder cells without VP-16 treatment were designated as the control group (con). The FAK inhibitor PF562271 was added to the culture medium of the feeder cells with or without VP-16 treatment, and the receptor cells were collected after a 10-day culture. Receptor cells located in the semipermeable filter insert were fixed with methanol and then stained with $0.1 \%$ crystal violet for $30 \mathrm{~min}$ at room temperature. After washing the wells with PBS, the cells on the upper surface of the membranes were imaged under a microscope. Finally, the cells were solubilized with $1 \mathrm{~mL}$ of $33 \%$ acetic acid and quantified by their absorbance at $570 \mathrm{~nm}$. We set the average $\mathrm{OD}_{570}$ of cells from control group to $100 \%$, and then determined the percentage of stimulated cells that were viable (attached) by comparing the average $\mathrm{OD}_{570}$ values of stimulated cells with the $\mathrm{OD}_{570}$ values of the nonstimulated cells.

\section{Western blot analysis}

Feeder cells from the third day of incubation were collected to analyze the protein level of caspase 3 . Feeder cells from the sixth day of incubation were collected to analyze the protein expression of iPLA. Receptor cells from the sixth day of incubation were collected for protein extraction for Western blot analysis of FAK and p-FAK. The collected SKOV3 cells (either receptor cells or feeder cells) grown in the co-culture system were collected by centrifugation after washing twice with cold PBS. The cell pellets were suspended in $100 \mu \mathrm{L}$ of radioimmunoprecipitation assay lysis buffer for $15 \mathrm{~min}$ at $4^{\circ} \mathrm{C}$ and then centrifuged at $13,000 \mathrm{rpm}$ for $15 \mathrm{~min}$. The supernatants containing the total protein extracts were collected. The concentrations of the proteins were determined using the BCA protein assay kit. Immunoblotting analysis was performed with $20 \mu \mathrm{g}$ of protein from each sample on a $12 \%$ sodium dodecyl sulfate (SDS) gel via polyacrylamide gel electrophoresis (PAGE). The electrophoresis was carried out initially at $80 \mathrm{~V}$ for $20 \mathrm{~min}$ followed by $160 \mathrm{~V}$ for 60-90 min. The proteins separated using SDS-PAGE were transferred onto PVDF membranes. The membranes were incubated for $2 \mathrm{~h}$ in blocking buffer $(10 \mathrm{mM}$ Tris $-\mathrm{HCl}$, $150 \mathrm{mM} \mathrm{NaCl}$, and $0.1 \%$ Tween-20) containing 5\% bovine serum albumin and then incubated with a primary antibody (1:800 or $1: 1,000)$ overnight. The membranes were then washed and incubated with a secondary antibody $(1: 2,000)$ at room temperature for $1 \mathrm{~h}$. Bands were visualized using an enhanced chemiluminescence Western blotting detection system (Tanon 4200).

\section{Measurement of $\mathrm{AA}$ and $\mathrm{PGE}_{2}$ levels}

The supernatants $(50 \mu \mathrm{L})$ were collected from the control and model groups in the Transwell system daily during the incubation period. Plasma samples from patients who underwent surgery alone and who underwent surgery and chemotherapy were presented as the control and chemotherapy groups, respectively. In vitro supernatants or patient plasma samples were thawed, and $\mathrm{PGE}_{2}$ and AA levels were analyzed using the human $\mathrm{PGE}_{2}$ ELISA kit and the human AA ELISA kit, respectively, according to the manufacturer's instructions. The absorbances were measured at a wavelength of $450 \mathrm{~nm}$.

\section{Statistical analysis}

All experiments were completed at least three times unless otherwise indicated. All the data were expressed as the mean \pm standard deviation. Statistical significance among 
groups was analyzed by one-way analysis of variance followed by Dunnett's multiple comparison test. A value of $P<0.05$ was considered statistically significant.

\section{Results}

\section{Levels of $A A$ and $P G E_{2}$ and the expression of FAK differ in the clinical} samples before and after chemotherapy

We first determined the levels of $\mathrm{AA}$ and $\mathrm{PGE}_{2}$ in patient plasma samples before and after chemotherapy. The level of $\mathrm{PGE}_{2}$ in patients who underwent chemotherapy was significantly higher than that in the control group, while the level of AA did not differ between the chemotherapy group and the control group. Also, the $\mathrm{PGE}_{2}$-to-AA ratio in plasma samples after chemotherapy was significantly higher than the ratio before chemotherapy (Figure 1A). This result suggested that chemotherapy might significantly influence the release of $\mathrm{AA}$ and $\mathrm{PGE}_{2}$. To understand whether the expression of FAK was altered after chemotherapy, we compared the expression levels of FAK and p-FAK in patient ovarian cancer tissues before and after chemotherapy (Figure 1B). The phosphorylation of FAK in the chemotherapy group was
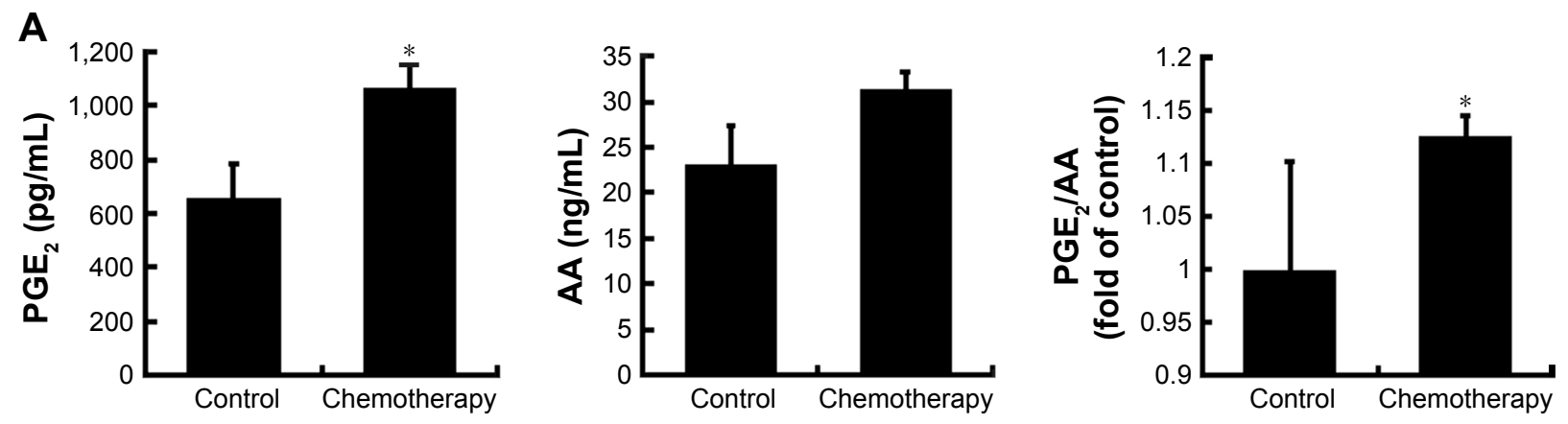

B

FAK

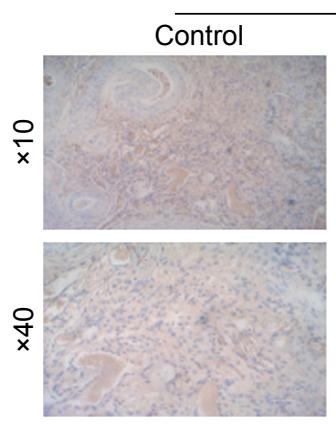

Chemotherapy

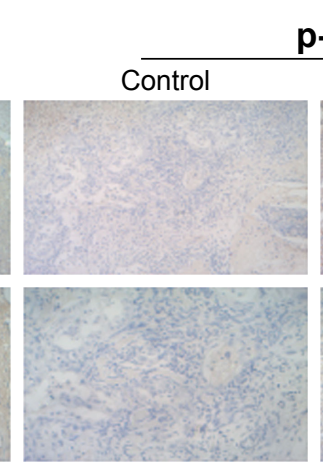

p-FAK

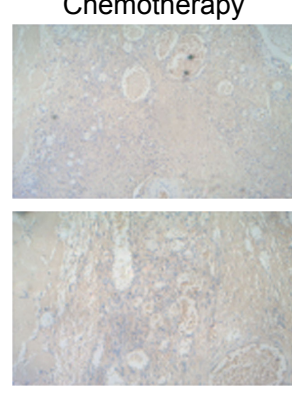

Chemotherapy
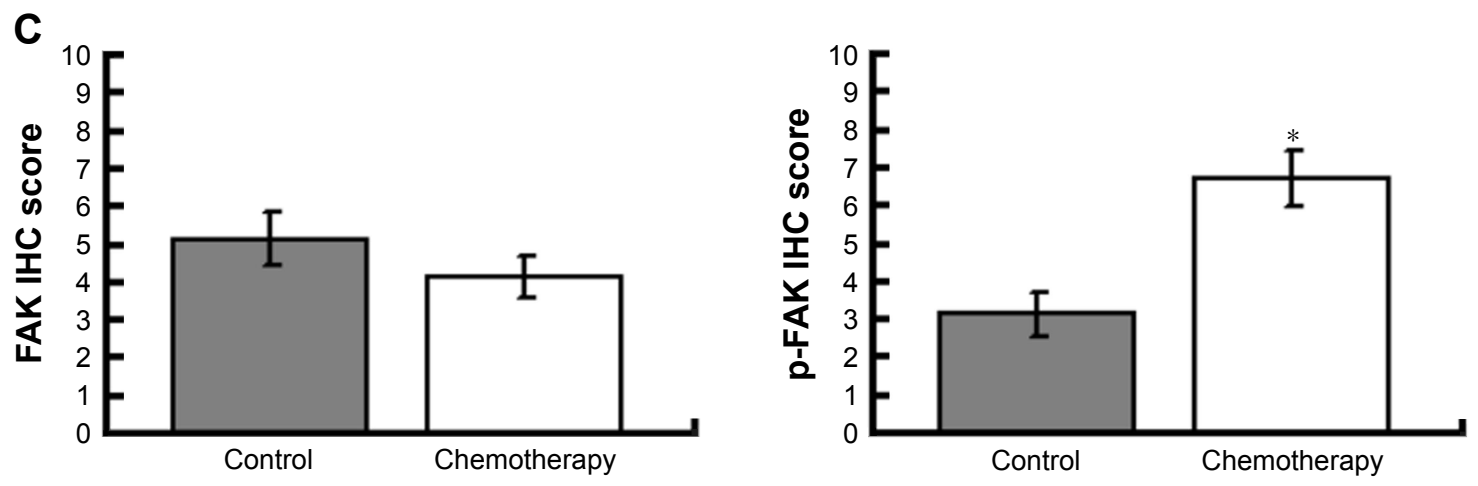

Figure I The PGE ${ }_{2}$ and $A A$ level and the expression of FAK in the clinical samples before and after chemotherapy.

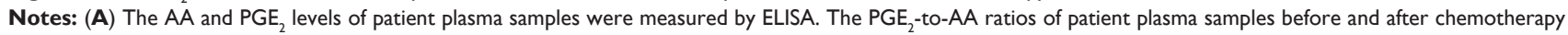
were compared. Three independent experiments were carried out. The results are expressed as the mean $\pm S D$ of three independent experiments, $* P<0.05$ compared with the control group. (B) Immunohistochemical staining of FAK and p-FAK in patient ovarian cancer samples before and after chemotherapy. (C) Levels of FAK and p-FAK were quantified according to immunohistochemical staining score.

Abbreviations: PGE $E_{2}$, prostaglandin $E_{2}$; AA, arachidonic acid; FAK, focal adhesion kinase; ELISA, enzyme-linked immunosorbent assay; SD, standard deviation; P-FAK, phosphorylated FAK; IHC, immunohistochemical. 
significantly higher than that in the control group (Figure 1C), indicating that the phosphorylation of FAK is associated with chemotherapy.

\section{VP-I6-induced apoptosis accelerates the repopulation of the SKOV3 cell line}

Accelerated repopulation causes cancer treatment failure. Researchers have observed that apoptotic tumor cells can stimulate the repopulation of tumors from a small number of surviving cells. In our study, we established a Transwell system for co-cultures of SKOV3 cells with the chemotherapy drug VP-16 or without the drug to examine whether VP-16 treatment can stimulate the growth of surviving ovarian cancer cells.

VP-16 treatment led to apoptosis of the feeder cells, and the result of the crystal violet staining assay of the receptor cells showed that compared with cells in the control group, receptor cells in the model (VP-16-treated) group showed significantly more proliferation under the microscope (Figure 2A). In addition, the cell viability was approximately 1.6-fold that of the control group (Figure 2B). This result confirmed that the VP-16-induced apoptosis can accelerate the repopulation of a small number of surviving SKOV3 cells.

\section{Caspase 3-activated iPLA ${ }_{2}-A A-P G E_{2}$ pathway is involved in the apoptosis- induced repopulation of SKOV3 cells}

Activated caspase 3, a key executioner in apoptosis, may be involved in growth stimulation of cells that survive chemotherapy. Figure 3 shows the activation of caspase 3 in feeder cells. The results showed that the VP-16-induced apoptosis can induce the expression of cleaved caspase 3 . We then attempted to identify the downstream factors of caspase 3 involved in generating growth-promoting factors in the dying cells, and focused on iPLA ${ }_{2}$. iPLA 2 , an enzyme of the AA metabolic network, is activated by caspase 3 cleavage. Caspase 3-mediated iPLA ${ }_{2}$ activation leads to increased production of AA, whose downstream eicosanoid derivatives (such as $\mathrm{PGE}_{2}$ ) have been implicated in stimulating tumor growth and stem cell proliferation.

To evaluate the potential involvement of the caspaseactivated $\mathrm{PLA}_{2}-\mathrm{AA}-\mathrm{PGE}_{2}$ pathway in the apoptosis-induced tumor cell proliferation, we examined the expression of iPLA $_{2}$ within the Transwell system. iPLA $_{2}$ levels in feeder cells treated with VP-16 were higher than those in the control group (Figure 3). In addition, we analyzed the AA and $\mathrm{PGE}_{2}$ levels of the supernatants collected daily from the Transwell system during the 10-day incubation. Over time, the amount of AA (Figure 4A) decreased, while the level of $\mathrm{PGE}_{2}$ (Figure 4B) increased. Significant differences between the model group and the control group appeared beginning at day 6 . We then calculated the content $\mathrm{PGE}_{2}$-to-AA ratio in the model group, and found that it increased in a time-dependent manner with significant differences from the control group appearing beginning at day 6 . The $\mathrm{PGE}_{2}$-to-AA ratio was 2.32 at day 10 (Figure 4C). These results confirmed our hypothesis that chemotherapy drug-induced apoptosis may lead to tumor cell repopulation and that the caspase $3-\mathrm{iPLA}_{2}-\mathrm{AA}-\mathrm{PGE}_{2}$ pathway may be involved in this process.

\section{$\mathrm{PEG}_{2}$ accelerates the repopulation of the SKOV 3 cell line through the activation of FAK}

To identify the downstream factors of $\mathrm{PGE}_{2}$, we focused on FAK, which is a key enzyme involved in the process of proliferation. Phosphorylation of FAK at tyrosine 397 (Tyr397) has been used as a marker of FAK activity. ${ }^{16}$ In addition, $\mathrm{PGE}_{2}$ treatment can increase FAK phosphorylation. ${ }^{13}$
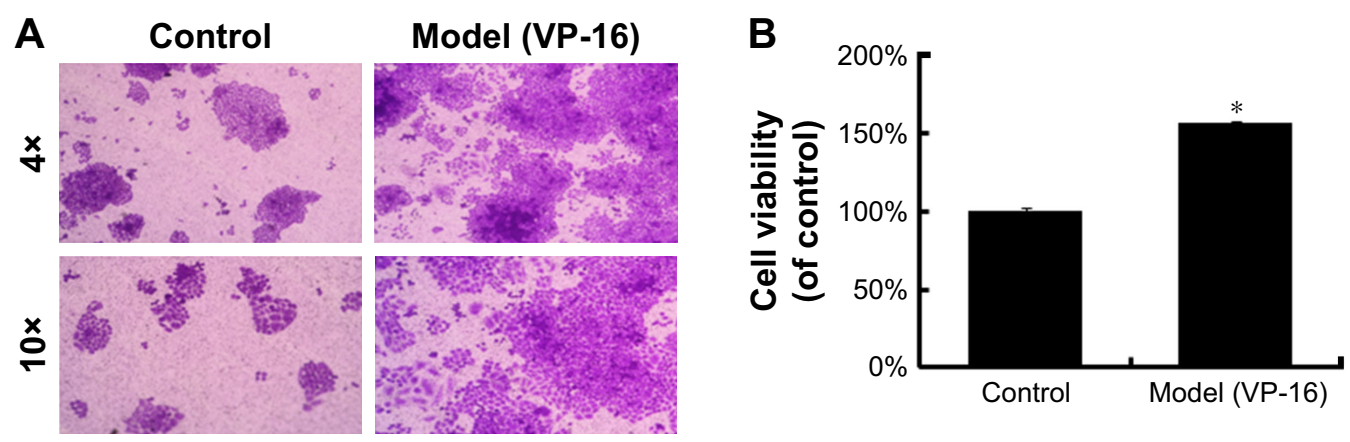

Figure 2 The influence of VP-16-induced apoptosis on the repopulation of SKOV3 cells.

Notes: (A) Crystal violet staining assay of the receptor cells in the control and model groups. (B) The stained cells of the two groups in (A) were solubilized with I $\mathrm{mL}$ of $33 \%$ acetic acid and quantified by the absorbance at $570 \mathrm{~nm}$. The results are expressed as the mean \pm SD of three independent experiments, $* P<0.05$ compared with the control group.

Abbreviations: VP-16, etoposide phosphate; SD, standard deviation. 

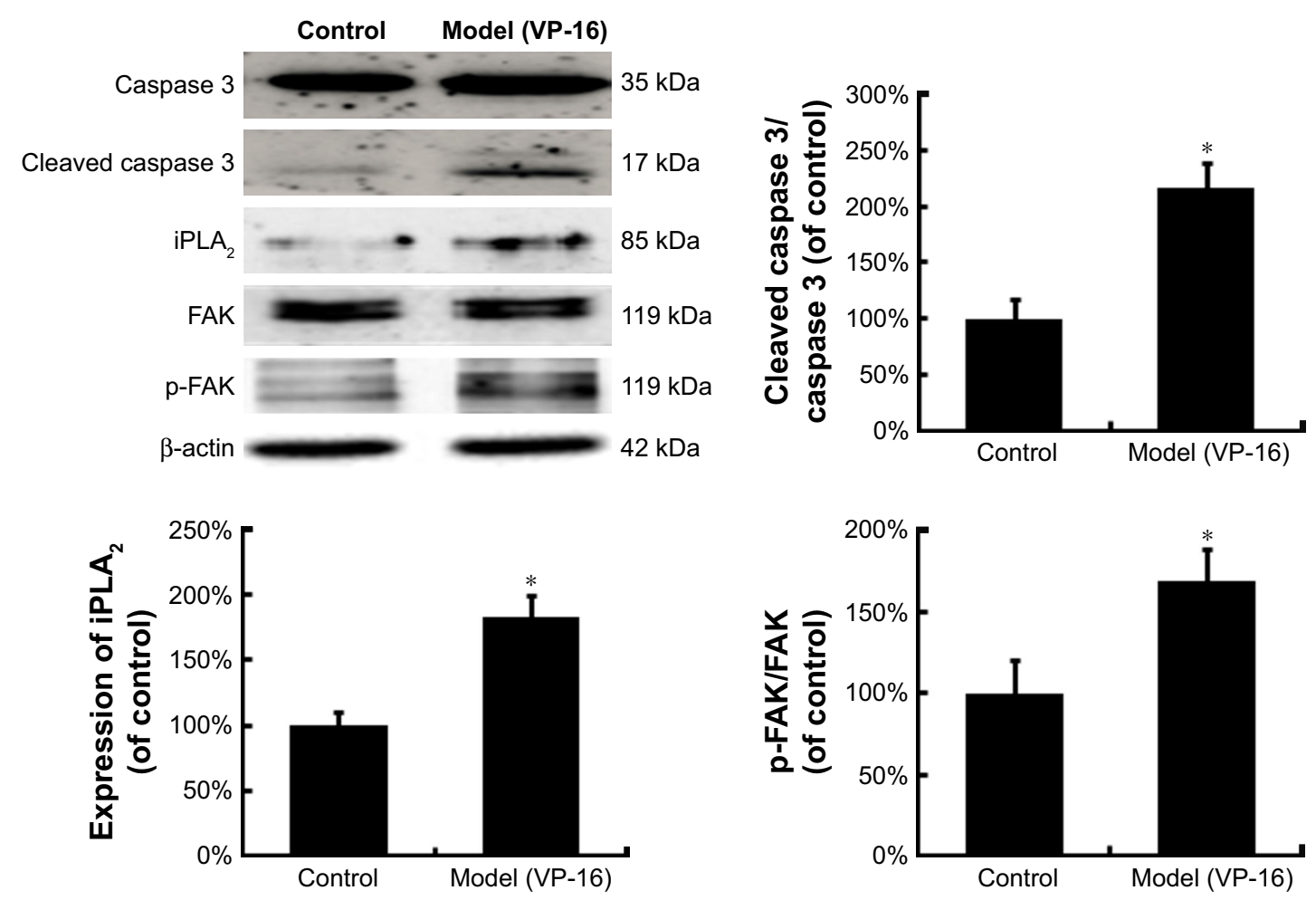

Figure 3 The protein expression of caspase 3, cleaved caspase 3, iPLA, FAK, and P-FAK in the model of the VP-I6-treatment-induced repopulation of SKOV3 cells. Notes: Western blot analysis of caspase 3, cleaved caspase 3, and iPLA expression in feeder cells and of FAK and p-FAK expression in receptor cells. Each bar represents the mean $\pm S D$ of three independent experiments, $n=3, * P<0.05$ compared with the control group.

Abbreviations: iPLA, cytosolic calcium-independent phospholipase $A_{2}$; FAK, focal adhesion kinase; p-FAK, phosphorylated FAK; VP-16, etoposide phosphate; SD, standard deviation.

To investigate the role of FAK in the pathways involved in the VP-16-induced repopulation, we measured the expression of FAK and p-FAK within the Transwell model on the 6th day of the incubation. The phosphorylation of FAK was significantly higher in the model group than in the control group, but the level of FAK remained unchanged
(Figure 3). Meanwhile, PF562271, an ATP competitor that inhibits the autophosphorylation of FAK, was added to the system. ${ }^{17}$ As shown in Figure 5, the addition of the FAK inhibitor partially reversed the VP-16-induced repopulation of SKOV3 cells. The expression of p-FAK was downregulated by PF 562271 but did not significantly influence the
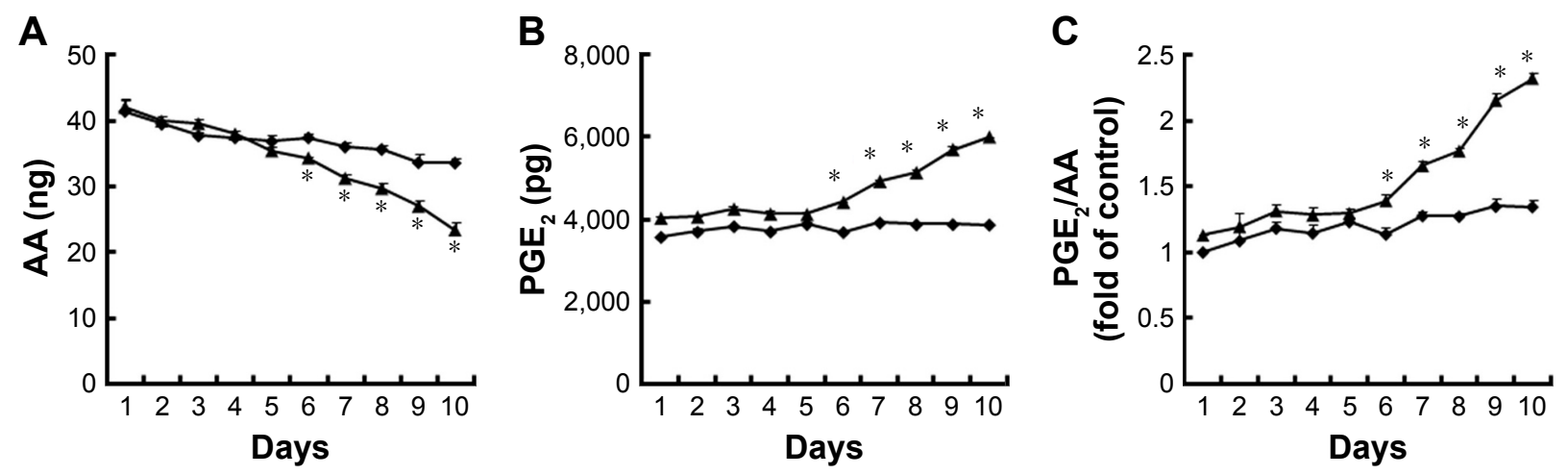

Control $\nrightarrow$ Model

Figure 4 The $A A$ and $P_{G E}$ levels within the Transwell system.

Notes: $(\mathbf{A}$ and $\mathbf{B})$ The $A A^{2}$ and $\mathrm{PGE}_{2}$ levels of the supernatants collected each day from the Transwell system during the I0-day incubation were measured by ELISA. (C) The $P G E_{2}$-to-AA ratios were calculated. Three independent experiments were carried out. The results are expressed as the mean $\pm S D$, $n=3$, $* P<0.05$ compared with the control group.

Abbreviations: $A A$, arachidonic acid; $\mathrm{PGE}_{2}$, prostaglandin $\mathrm{E}_{2}$; ELISA, enzyme-linked immunosorbent assay; SD, standard deviation. 

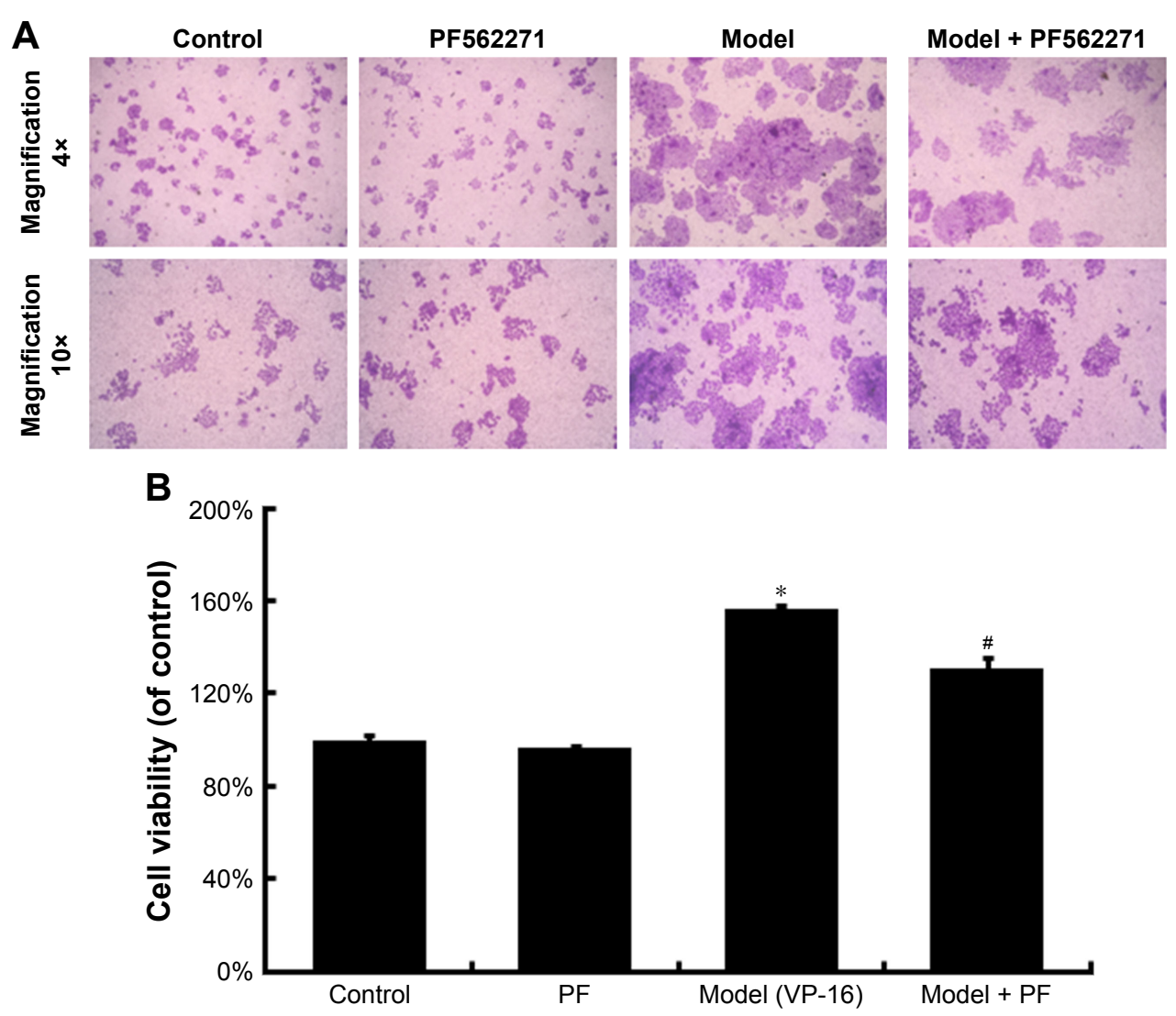

Figure 5 The influence of the FAK inhibitor PF56227I (PF) on the VP-I6-induced repopulation of SKOV3 cells.

Notes: (A) Crystal violet staining assay of the receptor cells with the FAK inhibitor PF56227I (PF) added to the system. (B) The stained cells of the four groups in A were solubilized with I mL of $33 \%$ acetic acid and quantified by the absorbance at $570 \mathrm{~nm}$. The results are expressed as the mean \pm SD of three independent experiments, $\mathrm{n}=3$, $* P<0.05$ compared with the control group, ${ }^{\# P}<0.05$ compared with the model group.

Abbreviations: FAK, focal adhesion kinase; VP-16, etoposide phosphate; SD, standard deviation.

expression of caspase 3 or iPLA 2 (Figure 6), suggesting that the phosphorylation of FAK may occur downstream of the caspase 3-iPLA 2 -AA-PGE 2 pathway and may influence the repopulation of SKOV3 cells.

\section{Discussion}

Treatment of ovarian cancer is based on the integration of surgery and chemotherapy. Chemotherapy plays a major role both in adjuvant treatment and in the care of patients with advanced disease. ${ }^{18}$ The standard of care in advanced epithelial ovarian cancer encompasses surgical staging and resection followed by administration of paclitaxel-platinumbased chemotherapy. ${ }^{19-21}$ Despite the high rates of overall tumor response associated with chemotherapeutics, many responders relapse within a short period after completing first-line therapy and require further systemic therapy. Recurrence is especially common in ovarian cancer. Patients who have recurrent ovarian cancer are rarely curable and often have only a short-term progression-free survival. ${ }^{3,4}$ Researchers have investigated the mechanism of cancer recurrence from various aspects, including apoptosis-related genes, chromosome aberration, angiogenesis, the cancer microenvironment, the immune systemancer stem cells. ${ }^{22}$ Cytotoxin chemotherapy is a common type of chemotherapy and is widely used in systemic therapy for cancer treatment. ${ }^{23}$ Previous studies have reported that radiotherapy or chemotherapy can lead to the measurable regrowth of tumor cells. ${ }^{7,8}$ In addition, $\mathrm{PGE}_{2}$ promotes the proliferation of cancer cells and stem cells in different types of cancer. In our study, $\mathrm{PGE}_{2}$ levels also increased in clinical samples. Thus, we propose that chemotherapy and the recurrence of ovarian cancer may be linked to cytotoxin chemotherapy drugs that induce $\mathrm{PGE}_{2}$ production, and despite the traditional role that chemotherapy has played in killing tumor cells, chemotherapy may have become a selective pressure that has led to the repopulation of ovarian cancer cells. In our study, chemotherapy was mechanistically determined to have two sides. On the one hand, chemotherapy killed cancer cells by inducing apoptosis, whereas it also caused the accelerated repopulation of a small number of surviving ovarian cancer 


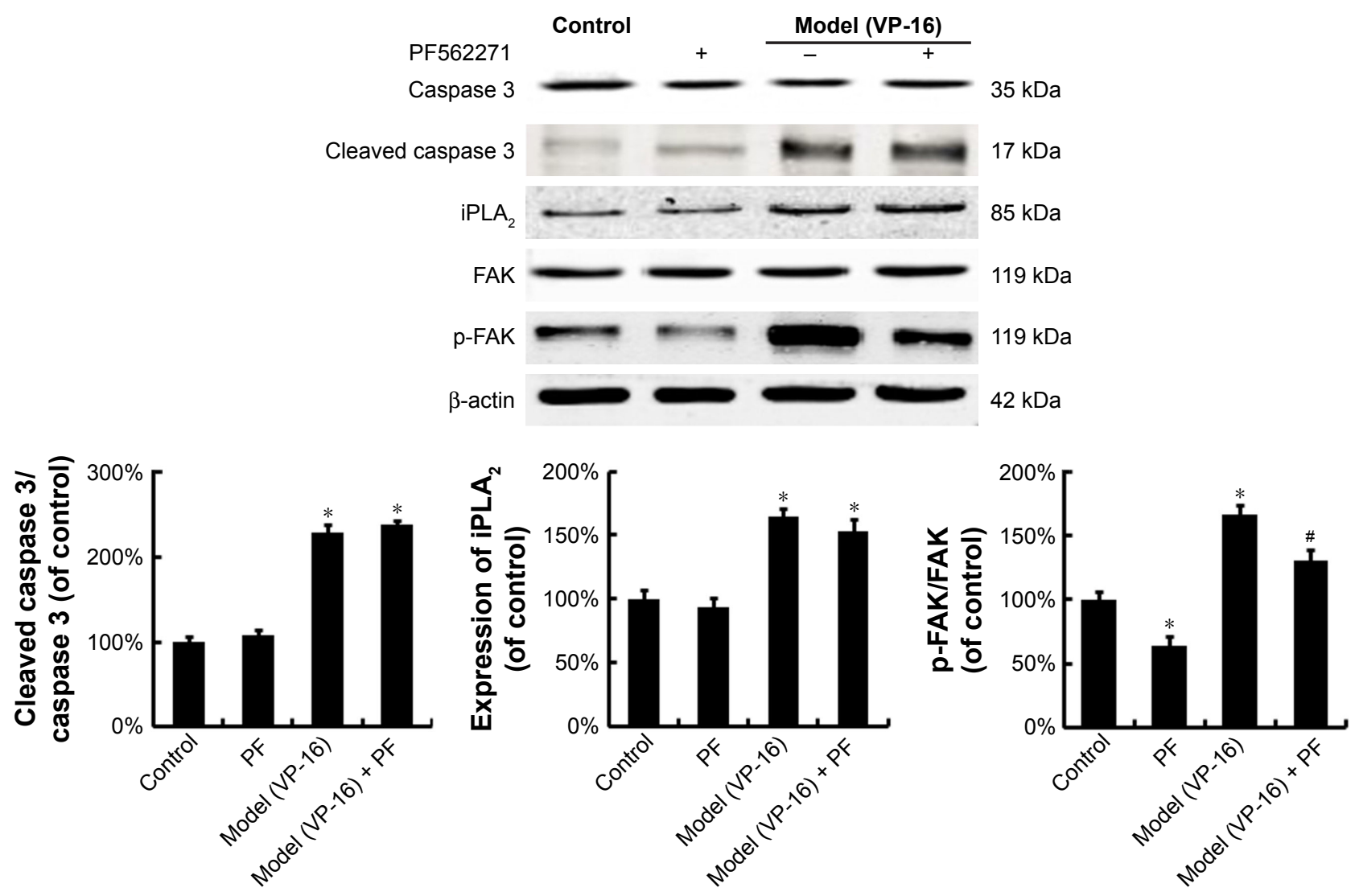

Figure 6 The influence of the FAK inhibitor PF56227I on the expression of FAK, P-FAK, caspase 3, and iPLA,

Notes: Western blot analysis of caspase 3, cleaved caspase 3, and iPLA, in feeder cells and of FAK and p-FAK in receptor cells with the FAK inhibitor PF56227I (PF) added to the system. Each bar represents the mean \pm SD of three independent experiments, ${ }^{*} P<0.05$ compared with the control group, ${ }^{\# P}<0.05$ compared with the model group.

Abbreviations: FAK, focal adhesion kinase; P-FAK, phosphorylated FAK; iPLA, cytosolic calcium-independent phospholipase $\mathrm{A}_{2}$; SD, standard deviation; VP-I6, etoposide phosphate.

cells through the caspase 3-activated iPLA 2 -AA-PGE 2 pathway followed by FAK phosphorylation. Thus, the application of an inhibitor that blocks this pathway after a chemotherapy course may become a solution to the high death rate in ovarian cancer patients.

The AA metabolic pathway has long been recognized to be correlated with the occurrence and development of various types of cancer. $\mathrm{PLA}_{2}$ and cyclooxygenase (COX) are rate-limiting enzymes in the AA metabolic pathway; these enzymes are important in the development and progression of a number of types of cancer. ${ }^{10,24} \mathrm{PLA}_{2}$ comprises a set of extracellular and intracellular enzymes. ${ }^{25}$ The various groups of PLA $\mathrm{P}_{2}$ can be mainly divided into three categories: secretory $\mathrm{PLA}_{2}$, cytosolic PLA 2 (cPLA $)$ and iPLA $_{2} \cdot{ }^{26,27}$ iPLA $_{2}$ plays a functional role in cellular signaling and AA release. ${ }^{28}$ Besides iPLA $_{2}$, which we studied, a major contribution of $\mathrm{cPLA}_{2}$ enzyme activity is also to mediate AA release. ${ }^{29}$ Downregulation of COX-2 expression leads to increased abundance of its principal metabolic product, $\mathrm{PGE}_{2}$, the pleiotropic effects of which appear to affect most, if not all, of the hallmarks of cancer. ${ }^{5,30} \mathrm{PGE}_{2}$ is a potent promoter of cell proliferation, motility, invasion, and angiogenesis. ${ }^{24}$ Similar results were obtained in our previous studies: celecoxib, a potent nonsteroidal anti-inflammatory drug, downregulates COX-2 protein expression in Lewis lung carcinoma cells. In addition, celecoxib increases the amount of AA and decreases the amount of $\mathrm{PGE}_{2}$ in the culture supernatant. AA plays an important role in $\mathrm{PGE}_{2}$ production, and in our study, the $\mathrm{PGE}_{2}$-to-AA ratio increased in a dose-dependent manner. ${ }^{31}$ In breast cancer cells, chemotherapy-induced apoptotic tumor cells use apoptosis to generate $\mathrm{PGE}_{2}$-stimulating signals to promote the repopulation of tumors. In this process, the few surviving cells that escape death after exposure to radiotherapy or chemotherapy can rapidly repopulate the badly damaged tumor by proliferating at a markedly accelerated pace via the caspase 3-PLA $-\mathrm{AA}_{2} \mathrm{PGE}_{2}$ pathway. ${ }^{5,6,8}$ In our study, we found that VP-16 treatment increased the amount of $\mathrm{PEG}_{2}$ and decreased the amount of $\mathrm{AA}$ in 
the culture supernatant. In addition, the $\mathrm{PGE}_{2}$-to-AA ratio which increased in a time-dependent manner suggested that caspase 3 cleavage-activated PLA $_{2}$ stimulated the release of AA. This stimulation ultimately triggered the production of $\mathrm{PGE}_{2}$ and confirmed the crucial role of the AA metabolic pathway (iPLA $\mathrm{A}_{2}-\mathrm{AA}-\mathrm{PGE}_{2}$ pathway) in the process of chemotherapy-induced cancer cell repopulation. It is worth to note that although Figure 4A showed increased manner of AA level in the patients after chemotherapy, there was no statistical significance between the two groups. The significantly increased $\mathrm{PEG}_{2}$-to-AA ratio was also consistent with our in vitro data.

As a key regulator of cellular signaling pathways that control cell proliferation and a protein downstream of $\mathrm{PGE}_{2}, \mathrm{FAK}$ is associated with proteins of the AA metabolic pathway. ${ }^{11,14,32-34}$ For example, exogenous $\mathrm{PGE}_{2}$ can increase FAK phosphorylation at Tyr397 in a concentration-dependent manner in HCC cells. At the same time, the selective COX-2 inhibitor celecoxib decreases FAK expression. These results implied that FAK phosphorylation may be regulated by the level of $\mathrm{PGE}_{2}{ }^{5,13}$ However, whether the chemotherapyinduced repopulation of ovarian cancer cells occurs through the activation of FAK remains unclear. In our study, FAK phosphorylation was found to increase in both the Transwell system that we established and in the patient tissue samples after chemotherapy. Addition of the FAK inhibitor PF562271 partially reversed the VP-16-induced repopulation of SKOV3 cells. The expression of p-FAK was downregulated by PF562271, while the inhibitor did not significantly influence the expression of caspase 3 or iPLA ${ }_{2}$, suggesting that FAK phosphorylation might be downstream of the caspase 3-iPLA $-\mathrm{AA}-\mathrm{PGE}_{2}$ pathway and may contribute to the repopulation of SKOV3 cells after chemotherapy. In addition, this confirmed our hypothesis: as chemotherapy leads to consistent changes in the microenvironment of ovarian cancer cells, the chemotherapy-induced dysregulation of the AA metabolic pathway may lead to abnormal activation of FAK and thus accelerate the repopulation of SKOV3 cells.

\section{Conclusion}

In summary, we determined that VP-16 treatment triggered the process of apoptosis, caspase 3 cleavage, and the activation of $\mathrm{iPLA}_{2}$, which stimulated the release of AA and ultimately triggered the production of $\mathrm{PGE}_{2}$. Activation of the AA metabolic pathway in a large number of dying cells stimulated the growth of a small number of surviving cells by the activation of FAK. In patient tissue and plasma samples, the level of $\mathrm{PGE}_{2}$ increased after chemotherapy, and the
$\mathrm{PGE}_{2}$-to-AA ratio after chemotherapy was significantly lower than that before chemotherapy. These results suggest that although chemotherapy induces cancer cell apoptosis, chemotherapy may also trigger the signals that ultimately lead to ovarian cancer recurrence.

\section{Acknowledgments}

Prof Jing Li, Fengying Guan, and Ming Zhang are acknowledged for their help in preparing the paper. This work was sponsored by the National Natural Science Foundation of China (81201804 and 81371681), the Science and Technology Department of Jilin Province (20160101215JC), the Opening Project of State Key Laboratory of Supramolecule Structure and Materials of Jilin University (SKLSSM 201605), and the Undergraduate Innovative Program of Jilin University (2015741091). Most of the experiments were carried out at the Nanomedicine Engineering Laboratory of Jilin Province and the Preclinical Pharmacology R\&D Center of Jilin Province.

\section{Disclosure}

The authors report no conflicts of interest in this work.

\section{References}

1. Ferlay J, Soerjomataram I, Dikshit R, et al. Cancer incidence and mortality worldwide: sources, methods and major patterns in GLOBOCAN 2012. Int J Cancer. 2015;136(5):E359-E386.

2. Bristow RE, Chang J, Ziogas A, Campos B, Chavez LR, Anton-Culver H. Impact of national cancer institute comprehensive cancer centers on ovarian cancer treatment and survival. J Am Coll Surgeons. 2015; 220(5):940-950.

3. Zhang H, Ge T, Cui X, et al. Prediction of advanced ovarian cancer recurrence by plasma metabolic profiling. Mol Biosyst. 2015;11(2): 516-521.

4. Salani R, Backes FJ, Fung MF, et al. Posttreatment surveillance and diagnosis of recurrence in women with gynecologic malignancies: Society of Gynecologic Oncologists recommendations. Am J Obstet Gynecol. 2011;204(6):466-478.

5. Wu L, Tannock IF. Repopulation in murine breast tumors during and after sequential treatments. Cancer Res. 2003;63(9):2134-2138.

6. Li F, Huang Q, Chen J, et al. Apoptotic cells activate the "phoenix rising" pathway to promote wound healing and tissue regeneration. Sci Signal. 2010;3(110):ra13.

7. Yom SS. Accelerated repopulation as a cause of radiation treatment failure in non-small cell lung cancer: review of current data and future clinical strategies. Semin Radiat Oncol. 2015;25(2):93-99.

8. Huang Q, Li F, Liu X, et al. Caspase 3-mediated stimulation of tumor cell repopulation during cancer radiotherapy. Nat Med. 2011;17(7): 860-866.

9. Yang P, Cartwright CA, Li J, et al. Arachidonic acid metabolism in human prostate cancer. Int J Oncol. 2012;41:1495-1503.

10. McLean GW, Carragher NO, Avizienyte E, Evans J, Brunton VG, Frame MC. The role of focal-adhesion kinase in cancer - a new therapeutic opportunity. Nat Rev Cancer. 2005;5(7):505-515.

11. Yoon H, Dehart JP, Murphy JM, Lim ST. Understanding the roles of FAK in cancer: inhibitors, genetic models, and new insights. $J$ Histochem Cytochem. 2015;63(2):114-128. 
12. Stokes JB, Adair SJ, Slack-Davis JK, et al. Inhibition of focal adhesion kinase by PF-562,271 inhibits the growth and metastasis of pancreatic cancer concomitant with altering the tumor microenvironment. Mol Cancer Ther. 2011;10(11):2135-2145.

13. Bai X, Zhang W, Liu N, et al. Focal adhesion kinase: important to prostaglandin E2-mediated adhesion, migration and invasion in hepatocellular carcinoma cells. Oncol Rep. 2009;21(1):129-136.

14. Sun BZ, Li J, Shao D, et al. Adipose tissue-secreted miR-27a promotes liver cancer by targeting FOXO1 in obese individuals. Onco Targets Ther. 2005;8:735-744.

15. Mao P, Smith L, Xie WF, Wang MD. Dying endothelial cells stimulate proliferation of malignant glioma cells via a caspase 3-mediated pathway. Oncol Lett. 2013;5:1615-1620.

16. Fong YC, Liu SC, Huang CY, et al. Osteopontin increases lung cancer cells migration via activation of the alphavbeta3 integrin/FAK/Akt and NF-kappaB-dependent pathway. Lung Cancer. 2009;64(3):263-270.

17. Yoon H, Choi YL, Song JY, et al. Targeted inhibition of FAK, PYK2 and BCL-XL synergistically enhances apoptosis in ovarian clear cell carcinoma cell lines. PLoS One. 2014;9(2):e88587.

18. Pignata S, Cannella L, Leopardo D, Pisano C, Bruni GS, Facchini G. Chemotherapy in epithelial ovarian cancer. Cancer Lett. 2011;303:73-83.

19. Kampan NC, Madondo MT, McNally OM, Quinn M, Plebanski M. Paclitaxel and its evolving role in the management of ovarian cancer. Biomed Res Int. 2015;2015:413076.

20. Nagano H, Tachibana Y, Kawakami M, et al. Patients with advanced ovarian cancer administered oral etoposide following taxane as maintenance chemotherapy. Case Rep Oncol. 2016;9(1):195-204.

21. Zhang SH, Huang Q. Etoposide induces apoptosis via the mitochondrialand caspase-dependent pathways and in non-cancer stem cells in Panc-1 pancreatic cancer cells. Oncol Rep. 2013;30(6):2765-2770.

22. Barker HE, Paget JT, Khan AA, Harrington KJ. The tumour microenvironment after radiotherapy: mechanisms of resistance and recurrence. Nat Rev Cancer. 2015;15(7):409-425.

23. Sonpavde G, Wang CG, Galsky MD, Oh WK, Armstrong AJ. Cytotoxic chemotherapy in the contemporary management of metastatic castrationresistant prostate cancer (mCRPC). BJU Int. 2015;116(1):17-29.
24. Li J, Li O, Kan M, et al. Berberine induces apoptosis by suppressing the arachidonic acid metabolic pathway in hepatocellular carcinoma. Mol Med Rep. 2015;12(3):4572-4577.

25. Zhao X, Wang D, ZhaoZ, et al. Caspase-3-dependent activation of calciumindependent phospholipase A2 enhances cell migration in non-apoptotic ovarian cancer cells. J Biol Chem. 2006;281(39):29357-29368.

26. Lee C, Park DW, Lee J, et al. Secretory phospholipase A2 induces apoptosis through TNF-alpha and cytochrome c-mediated caspase cascade in murine macrophage RAW 264.7 cells. Eur J Pharmacol. 2006;536(1-2):47-53.

27. Castellone MD, Teramoto H, Williams BO, Druey KM, Gutkind JS. Prostaglandin E2 promotes colon cancer cell growth through a Gs-axinbeta-catenin signaling axis. Science. 2005;310(5753):1504-1510.

28. Song K, Zhang X, Zhao C, Ang NT, Ma ZA. Inhibition of $\mathrm{Ca}^{2+}$-independent phospholipase A2 results in insufficient insulin secretion and impaired glucose tolerance. Mol Endocrinol. 2005;19(2):504-515.

29. Dan P, Rosenblat G, Yedgar S. Phospholipase A2 activities in skin physiology and pathology. Eur J Pharmacol. 2012;691(1-3):1-8.

30. Greenhough A, Smartt HJ, Moore AE, et al. The COX-2/PGE2 pathway: key roles in the hallmarks of cancer and adaptation to the tumour microenvironment. Carcinogenesis. 2009;30(3):377-386.

31. Zhang M, Xu ZG, Shi Z, et al. Inhibitory effect of celecoxib in lung carcinoma by regulation of cyclooxygenase-2/cytosolic phospholipase $\mathrm{A}(2)$ and peroxisome proliferator-activated receptor gamma. Mol Cell Biochem. 2011;355(1-2):233-240.

32. Kajimoto M, Ichiyama T, Ueno Y, Shiraishi M, Hasegawa M, Furukawa S. Enhancement of activated $\beta 1$-integrin expression by prostaglandin E2 via EP receptors in isolated human coronary arterial endothelial cells: implication for the treatment of Kawasaki disease. Inflamm Res. 2009; 58(4):224-228.

33. Gervais FG, Thornberry NA, Ruffolo SC, et al. Caspases cleave focal adhesion kinase during apoptosis to generate a FRNK-like polypeptide. J Biol Chem. 1998;273(27):17102-17108.

34. Wang S, Ke Y, Lu G, et al. Vasculogenic mimicry is a prognostic factor for postoperative survival in patients with glioblastoma. J Neurooncol. 2013;112(3):339-345.
OncoTargets and Therapy

\section{Publish your work in this journal}

OncoTargets and Therapy is an international, peer-reviewed, open access journal focusing on the pathological basis of all cancers, potential targets for therapy and treatment protocols employed to improve the management of cancer patients. The journal also focuses on the impact of management programs and new therapeutic agents and protocols on

\section{Dovepress}

patient perspectives such as quality of life, adherence and satisfaction The manuscript management system is completely online and includes a very quick and fair peer-review system, which is all easy to use. Visit http://www.dovepress.com/testimonials.php to read real quotes from published authors. 